\title{
THERMAL PROPERTIES MEASUREMENTS USING LASER FLASH TECHNIQUE AT CRYOGENIC TEMPERATURE
}

\author{
G. Penco, D. Barni, P. Michelato, C. Pagani, INFN Milano-LASA, Segrate (MI), Italy
}

\begin{abstract}
In the frame of the Italian TRASCO program, we have studied the possibility to stiff SC cavities by deposition of thermal sprayed copper on thin niobium. We have investigated the use of the Laser Flash technique to test the thermal conductivity in the normal direction of deposited copper with the Pure Coat technique. The use of $\mathrm{Au}-\mathrm{Fe} / \mathrm{Cromel} \mathrm{P}$ thermocouples as temperature sensors, motivated by the need to adapt the classical technology from room temperature to the cryogenic environment, allowed the thermal transient study. A great effort has been spent to develop data analysis procedures, in order to reduce noise and heat dissipation effects. The measurements have demonstrated the reliability of the apparatus and the results confirm the interest in the copper spraying stiffening for the SC cavities.
\end{abstract}

\section{INTRODUCTION}

In the recent development of high field SC cavities, a great effort has been spent to study stiffening methodologies for pulsed operation. The design of all the new cavities takes into account the action of external factors, like Lorentz forces and microphonics, that affect the cavity shape and consequently the field amplitude and phase. The reference stiffening scheme is that developed for the TESLA Test Facility (TTF) cavities by the TESLA Collaboration [1]. It consists of a niobium ring welded between adjacent cells at the radius that minimize the Lorentz force detuning.

In the framework of the Italian TRASCO project [2] for nuclear waste transmutation, we studied the possibility of improving the cavity stiffness through the combination of a TTF-like ring, close to the iris region, and a copper coating at the equator. Tests on samples showed that the thermal spray technique called "Pure Coat" [3] potentially gives the best coating properties.

In principle, a high thermal conductivity copper should allow a thicker cavity wall for the same temperature drop and also the Kapitza resistance at the He interface should be lower. Unfortunately all the copper deposition techniques investigated so far [4] are worse than the ideal case, mainly because their microstructure presents a lot of oxide inclusions and porosities that reduce both the thermal conductivity and the Young module. As a consequence, it was necessary to investigate the thermal properties of different copper coatings, at cryogenic temperature, to test and verify their compatibility with the SC cavities technologies.

The most used way to measure the thermal conductivity consists in generating a thermal gradient in a sample and measuring the stationary temperature distribution.
In the case of film samples or thin coatings, as the thermal sprayed copper, it is often interesting to measure the thermal properties in the normal direction, in order to investigate the heat propagation through these materials, which often present a strong anysotropic microstructure.

For this purpose in the last decades a technique called Thermal Wave Interferometry (TWI) has been studied. TWI studies the propagation of the thermal waves on a sample and the interference effects due to a sort of reflection of the incident wave on the opposite surface [5]. Since a temperature sensor kept directly in contact with the rear surface would introduce a sensible variation on the experiment, the phase of the reflected thermal wave, the most important parameter, can be measured only by an IR detector. In this experimental condition it is very critical to adapt this technique to the cryogenic environment. For these reasons it has been studied an alterative technique to extend the thermal conductivity investigation to cryogenic temperatures.

\section{LASER FLASH}

The Laser Flash technique is based on the measure of the thermal transient of the rear surface of the sample when a pulsed laser illuminates the front: in this way it is possible to avoid interferences between the thermal sensor and the heat source [6]. The physical model of the Laser Flash measurement supposes to have a single pulsed heat source (delta like), for example a laser shot, on the sample front surface. The study of the thermal transient of the rear surface provides the desired thermal information. Assuming that the laser beam fits the sample front surface uniformly, the heat source generates a one direction thermal gradient that can be described with a onedimensional differential equation.

Applying the correct boundary conditions the solution of the Fourier equation for heat diffusion is:

$T(x, t)=T_{\text {end }}\left[1+2 \sum_{n=1}^{\infty}(-1)^{n} e^{-\frac{n^{2} \pi^{2} D}{L^{2}} \cdot t} \cdot \cos \left(\frac{n \pi(L-x)}{L}\right)\right]$

where $T_{\text {end }}$ is the sample total temperature variation, $D$ the thermal diffusivity and $L$ the sample thickness. Normalizing equation (1) relative to the rear surface with respect to $T_{\text {end }}$, we have

$$
V(\tau)=1+2 \sum_{n=1}^{\infty}(-1)^{n} \cdot e^{-n^{2} \cdot \tau}
$$

where $\tau=\pi^{2} \cdot t \cdot D / L^{2}$. Eq. (2) provides the final temperature of the back surface at the time $\tau$. 
The first advantage of the formulation presented in eq. (2) is that the normalized solution is independent from the energy transmitted to the sample, which is often not completely known, for example for reflection effects and inhomogeneities in the laser spot shape. In addition the analysis needs to know only a normalized temperature measurement, and no absolute measurements, so it is enough that the temperature sensor has a linear response to the temperature variation.

Here we present an extension of the Laser Flash technique to the cryogenic environments that uses a standard temperature sensor in direct contact with the rear surface.

First of all the fast variation of the temperature, due to the very low specific heat and high thermal conductivity of the sample, implies that the sensor and the data acquisition system should be fast enough to resolve the thermal transient without perturbations. For this purpose the sensor needs to have a much lower thermal inertia than the sample and it needs to be in close contact with the sample itself to avoid any thermal resistance affecting the results. According to these sensor requirements, we have chosen to measure the thermal transient with very small thermocouples $\mathrm{Au}(0.007 \% \mathrm{Fe}) /$ Cromel P, that are very sensible at cryogenic temperature and have a very small thermal mass. At cryogenic temperatures it is necessary to induce small temperature variations to remain in the approximation of constant thermal diffusivity. The thermocouple voltage results in the microvolts range and, therefore, must be amplified. To test the time resolution limit of the experimental setup a laser single shot has been sent on the thermocouple alone at room temperature, and we measured a response time of about $10 \mu \mathrm{s}$, that corresponds to the chosen amplifier bandwidth. In this case the time resolution limit is independent from the temperature sensor itself.

\subsection{Experimental Setup}

For the cryogenic temperature measurements the test sample is fitted inside a vacuum insulated system $\left(10^{-3}\right.$ mbar) to fulfil the adiabatic condition and to avoid convection effects.

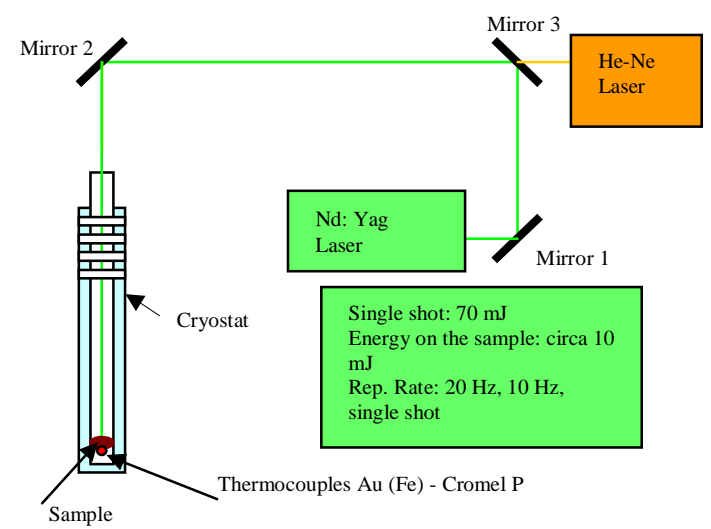

Figure 1 Experimental Setup. Because of the high intensity of the laser we use a He-Ne laser exactly superimposed to the Nd-YAG for alignment
We have used is a Niodium Laser (Yag laser) as the heat source. The laser beam is driven in the cryostat straightly on the test sample, as it is shown in Figure 1.

\subsection{Test bench on OFHC copper}

To test the accuracy of the Laser Flash measurements we have performed the first tests on a well-known copper sample (OFHC: Oxygen Free High Conductivity), whose thermal properties are known from literature. This test bench is also useful because it allows the comparison between the excellent thermal properties of OFHC and the results obtained from the Pure Coat coating.

The thermocouples voltage signal during the transient, at $300 \mathrm{~K}$ and at $77 \mathrm{~K}$ is only a few microvolts, due to the high energy fraction reflected by the sample and to the thermal capacitance of copper. The electromagnetic noise in the acquisition system is about $1 \mu \mathrm{V}$ and is comparable to the signal, so it is critical to extract the sample exact thermal behaviour. To simplify the data analysis we have used a train of laser shots (at the laser repetition rate) on the sample and we have acquired the whole thermal transient induced by the series of laser shots. The thermal transient of each single shot is then extracted and normalized to 1 , using the pretrigger data and the flat region (stationary temperature) after the transient as references. Data from all shots are then smoothed. The smoothed data need to be compared with the theoretical function of the transient. Using this data analysis procedure we get a final thermal transient (Fig. 2) with a resolution of about $0.1 \mu \mathrm{V}$, starting with a thermoelectric signal in which the noise is dominant.

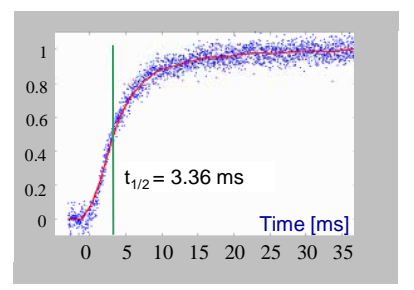

Figure 2: Final signal after filtering. The vertical line indicates the time needed to reach the $50 \%$ of the final temperature raise.

The choice of the repetition rate of the laser beam must take in account the foreseen raise time, in order to reach the stationary increase before the arrival of the following shot. In addition we have found that the most significant contribution to the noise is at $50 \mathrm{~Hz}$. Setting the laser frequency at $20 \mathrm{~Hz}$ this noise component is about cancelled by the smoothing between two consecutive thermal transients.

The study of the transient function shape is made by evaluating the thermal diffusivity from eq.(2) as a function of time. In the ideal case all these values of thermal diffusivity should be perfectly equal, but in a real experimental setup we have to take in account some perturbative effects. The first important effect is the nonuniform heating of the sample that breaks the one- 
dimensional assumption. For example, we report in Fig.3 the behaviour of the function $\mathrm{V}(\mathrm{t})$ in the ideal case of a central heater region (and a colder central region).

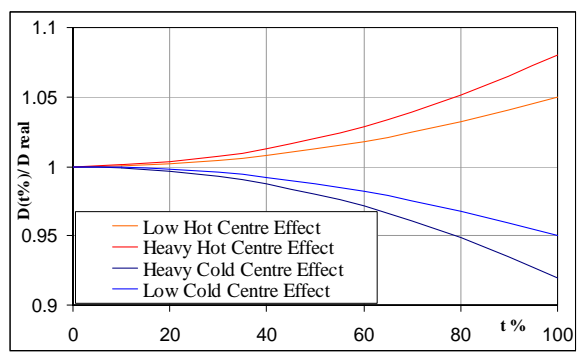

Figure 3. Behaviour of the thermal diffusivity in presence of hot/cold centre effect for fractions of the raise time

In presence of a hot (or cold) centre effect the sample heating results faster (or slower) than the ideal case and so the thermal diffusivity is overestimated (or underestimated) during the transient [7]. When the systematic errors are identified, it is possible to fit the data and to extrapolate the value at time $\tau=0$. The experimental apparatus that we have used does not show any of the anomalies presented in Fig. 3, but probably a combination of both and in addition there may be a combination of several other second order effects (for example the irregular shape of the laser spot, the irregular superposition between the laser spot and the sample, the deviation from the ideal adiabatic condition, and so on). For this reason it is useful to calculate the thermal diffusivity from eq. (2) by using different percentage of the raise time and then averaging the different values obtained [7]. The plot of these results, shown in Fig. 4, allows the identification of their confidence or indicates if some significant perturbation has occurred during the measurements. Table 1 summarizes the results on the OFHC copper $\left(D_{\exp }\right.$ is the experimental value and $D_{T h}$ the value found in literature):

Table 1. OFHC copper samples.

\begin{tabular}{|l|l|l|}
\hline $\mathbf{T}[\mathbf{K}]$ & $\mathbf{D}_{\mathbf{T h}}\left[\mathbf{c m}^{2} / \mathbf{s}\right]$ & $\mathbf{D}_{\text {Exp }}\left[\mathbf{c m}^{2} / \mathbf{s}\right]$ \\
\hline 300 & 1.14 & $1.20 \pm 0.05$ \\
\hline 77 & 2.80 & $2.56 \pm 0.28$ \\
\hline
\end{tabular}

\subsection{Pure Coat Samples}

After the test bench measurements on the OFHC copper we have measured a Pure Coat sample at $300 \mathrm{~K}$ and at $77 \mathrm{~K}$, obtaining the results reported in Tab. 2

Table 2. Pure coat samples.

\begin{tabular}{|c|c|}
\hline $\mathbf{T}[\mathbf{K}]$ & $\mathbf{D}_{\text {Exp }}\left[\mathbf{c m}^{2} / \mathbf{s}\right]$ \\
\hline 300 & $0.47 \pm 0.02$ \\
\hline 77 & $0.74 \pm 0.05$ \\
\hline
\end{tabular}

The experimental errors presented in Tab. 1 and 2 are calculated as standard deviation with respect to the average value of the thermal diffusivity, which is obtained from a weighted average of the values calculated for each fraction of the time raise (Figure 4):

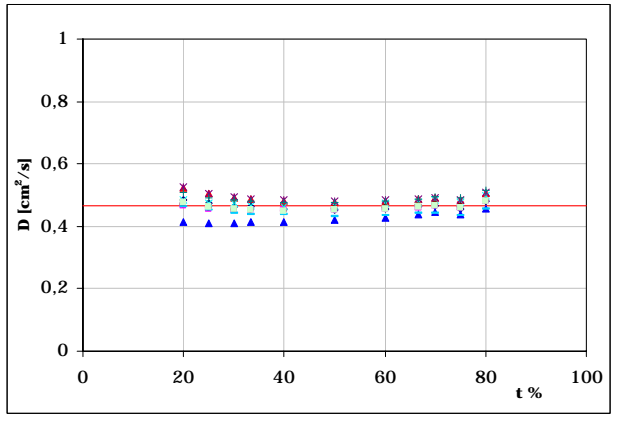

Figure 4 Values of thermal diffusivity calculated from eq. (2) using different percentages (t\%) of the raise time. The good data correlation and the constant trend suggests that the filter procedure reduces the perturbative effects.

Other Pure Coat samples have been measured, after annealing in ultra-vacuum $\left(10^{-7} \mathrm{mbar}\right)$ to improve their thermal properties (Table 3).

Table 3. Pure Coat annealed samples.

\begin{tabular}{|c|c|}
\hline $\mathbf{T}[\mathbf{K}]$ & $\mathbf{D}_{\text {Exp }}\left[\mathbf{c m}^{2} / \mathbf{s}\right]$ \\
\hline 77 & $1.27 \pm 0.07$ \\
\hline 7.0 & $86.1 \pm 9.1$ \\
\hline 5.5 & $112 \pm 9.8$ \\
\hline 4.2 & $200 \pm 18$ \\
\hline
\end{tabular}

The data analysis of measurements below $10 \mathrm{~K}$ is easier because the low thermal capacity produce higher temperature variation.

\section{CONCLUSION}

We have demonstrated that the laser flash technique can be used at cryogenic temperatures to investigate thermal properties. Results on annealed sprayed copper are interesting for proton cavities stiffening, having thermal properties closed to the requirements.

\section{REFERENCES}

[1] B. Aune et al., "Superconducting TESLA Cavities", Phys. Rev. ST-AB, Vol. 3, 092001 (2000).

[2] C.Pagani et al." Status of the High Energy SC Linac for the TRASCO program", these proceedings.

[3] G.Penco , Thesis, University of Milano 2000.

[4] S. Bousson et al., "SRF cavity stiffening by thermal spraying", in Proceedings of the EPAC 2000, Vienna, Austria, p. 2043.

[5] C.A. Bennett, Jr., and R.R. Patty, "Thermal wave interferometry: a potential application of the photoacustic effect”, Applied Optics, Vol.21, n ${ }^{\circ} 1,1982$.

[6] W.J. Parker, R.J. Jenkins, C.P. Butler, G.L. Abbot, "Flash Method of Determing Thermal Diffusivity, Heat Capacity and Thermal Conductivity", J. Appl. Phys., 32, 1679-84, 1964.

[7] J.A. Mackay, J.P. Schriempf, "Corrections for non uniform source heating errors in flash method thermal diffusivity measurements", J. Appl. Phys., 47, 1668-1671, 1971. 